\title{
Correction to: MiR-26a regulated adipogenic differentiation of ADSCs induced by insulin through CDK5/FOXC2 pathway
}

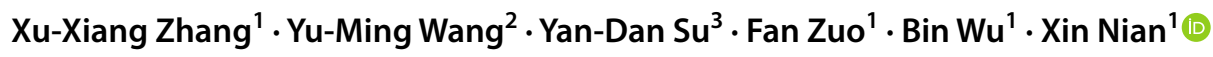

Published online: 22 June 2021

๑) Springer Science+Business Media, LLC, part of Springer Nature 2021

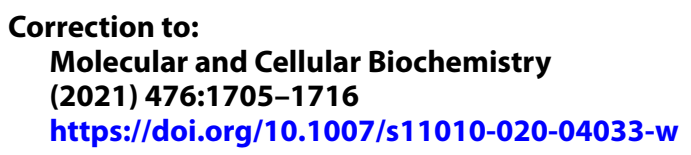

The e-mail address of the corresponding author was published incorrectly. The correct e-mail address is provided in this correction.

Publisher's Note Springer Nature remains neutral with regard to jurisdictional claims in published maps and institutional affiliations.

The original article can be found online at https://doi.org/10.1007/ s11010-020-04033-w.

\section{Xin Nian}

nianxinkm@hotmail.com

1 Department of Endocrinology, First Affiliated Hospital of Kunming Medical University, Kunming 650032,

Yunnan Province, People's Republic of China

2 Department of Clinical Laboratory, Second Affiliated Hospital of Kunming Medical University, Kunming 650032, Yunnan Province, People's Republic of China

3 Department of Clinical Laboratory, First Affiliated Hospital of Kunming Medical University, Kunming 650032,

Yunnan Province, People's Republic of China 